\title{
Almost Sure Relative Stability of the Overshoot of Power Law Boundaries
}

\author{
R.A. Doney ${ }^{1}$ and R.A. Maller ${ }^{2,3}$
}

Received July 13, 2005; revised November 16, 2005

\begin{abstract}
We give necessary and sufficient conditions for the almost sure relative stability of the overshoot of a random walk when it exits from a two-sided symmetric region with curved boundaries. The boundaries are of power-law type, $\pm r n^{b}, r>0, n=1,2, \ldots$, where $0 \leq b<1, b \neq 1 / 2$. In these cases, the a.s. stability occurs if and only if the mean step length of the random walk is finite and non-zero, or the step length has a finite variance and mean zero.
\end{abstract}

KEY WORDS: Random walk; Curved boundaries; Overshoot of power-law boundaries.

2000 MSC Subject Classifications: Primary: 60J15; 60F15, 60K05, 60G40; Secondary: 60F05.

\section{INTRODUCTION}

This paper continues the investigation begun in Ref. 3 of the asymptotic behaviour of the overshoot of a random walk when it exits from a two-sided symmetric region with curved boundaries of power-law form. In what follows, $S=\left(S_{n}, n \geqslant 0\right), S_{0}=0$, will denote the random walk with step size $X_{n}=S_{n}-S_{n-1}$ (sometimes we write $X(n)$ ), which is assumed to be non-degenerate with distribution function $F$, and $b$ will be a constant in the range $[0,1)$. We define the exit time

$$
T_{r}=\min \left\{n \geqslant 1:\left|S_{n}\right|>r n^{b}\right\}, \quad r>0
$$

\footnotetext{
${ }^{1}$ Department of Mathematics, University of Manchester, Manchester, M60 1QD, UK; E-mail: rad@maths.manchester.ac.uk

${ }^{2}$ School of Finance and Applied Statistics, Australian National University, Canberra, ACT, Australia; E-mail: Ross.Maller@anu.edu.au

${ }^{3}$ To whom correspondence should be addressed.
} 
(with $T_{r}=\infty$ if $\left|S_{n}\right| \leq r n^{b}$ for all $n \geqslant 1$ ), and the overshoot by

$$
O_{r}:=\left|S_{T_{r}}\right|-r T_{r}^{b} .
$$

Our aim is to find necessary and sufficient conditions for the overshoot to be almost surely (a.s.) asymptotically small as compared to the boundary, viz, for

$$
\frac{O_{r}}{r T_{r}^{b}}=\frac{1}{r}\left(\frac{\left|S_{T_{r}}\right|}{T_{r}^{b}}-r\right) \stackrel{\text { a.s. }}{\rightarrow} 0 \quad \text { as } r \rightarrow \infty ;
$$

we refer to this as almost sure relative stability of the overshoot.

For the case $0 \leqslant b<1, b \neq 1 / 2$, it was shown in Refs. 3 and 4 (see also Ref. 5 for the case $0<b<1 / 2$ and Ref. 9 and 6 for the case $b=0$ ) that the in probability version of (1.3) can occur in two and only two situations; $S$ has to be relatively stable, in the sense that $S_{n} / c_{n} \stackrel{P}{\rightarrow} \pm 1$ for some positive norming sequence $c_{n}$, or else, if $b<1 / 2, S$ must belong to the domain of attraction of the Normal distribution, without centering. (We write $S \in R S$ or $S \in D_{0}(N)$.) Moreover, in case $b=0$ it was also shown in Ref. 6 that a necessary and sufficient condition (NASC) for the a.s. result (1.3) is:

$$
\text { either } E X^{2}<\infty \text { and } E X=0 \text {, or } 0<|E X| \leq E|X|<\infty \text {. }
$$

In the present paper, we generalise this result to the case of curved (power law) boundaries. This is not a straightforward exercise since the techniques required to deal with almost sure results for non-constant boundaries have not previously been worked out, and it was not at all obvious that we could expect such a clearcut equivalence as in (1.4). (Nevertheless, our methods rely on some basic relationships worked out in Refs. 3 and 4.) As it turns out, we do get a very easily interpretable answer. The dichotomy in (1.4) essentially extends to our situation, and its simplicity augers well for possible applications of the result in statistics (especially, sequential analysis) and elsewhere.

As another application, we provide in Proposition 5 below an alternative derivation of a key result in Ref. 10 concerning the limsup behaviour of the random walk.

Theorem 1. If $0 \leqslant b<1$ and $b \neq \frac{1}{2}$, the following are equivalent:

$$
\begin{array}{cc}
(1.4) & \text { for } 0 \leqslant b<1 / 2 \\
0<|E X| \leq E|X|<\infty & \text { for } 1 / 2<b<1
\end{array}
$$




$$
T_{\mathrm{r}} \stackrel{\text { a.s. }}{<} \infty \quad \text { for all } r>0 \text { and } \quad \frac{O_{r}}{r T_{r}^{b}} \stackrel{\text { a.s. }}{\rightarrow} 0 \quad \text { as } r \rightarrow \infty
$$

and

(iii) $T_{r} \stackrel{\text { a.s. }}{<} \infty$ for all $r>0$ and $\limsup _{r \rightarrow \infty} \frac{\left|S_{T_{r}}\right| \text { a.s. }}{r T_{r}^{b}}<$ for some $L \in(1, \infty)$.

Remark 2. From Theorem 3.1 in Ref. 6, we can read off two more probabilistic conditions which are also equivalent to (1.4) and hence to (1.5)-(1.7) for the values of $b$ specified; with $S_{n}^{*}=\max _{1 \leq r \leqslant n}\left|S_{r}\right|$ and $\left|X_{n}^{(1)}\right|=\max _{1 \leq r \leqslant n}\left|X_{r}\right|$ they are

$$
\lim _{n \rightarrow \infty} \frac{S_{n}^{*}}{\left|X_{n}\right|} \stackrel{\text { a.s. }}{=} \infty
$$

and

$$
\lim _{n \rightarrow \infty} \frac{S_{n}^{*}}{\left|X_{n}^{(1)}\right|} \stackrel{\text { a.s. }}{=} \infty \text {. }
$$

We will also need an analytic condition equivalent to (1.4) which can be found in Lemma 4.2 of Ref. 6; to state it we need some notation. We write $X$ for a generic step in the random walk, and put, for $x>0$,

$$
\begin{aligned}
& G(x)=P(|X|>x), \quad U(x)=\int_{0}^{x} 2 y G(y) d y, \\
& A(x)=E((X \wedge x) \vee(-x)), \quad k(x)=x^{-2} U(x)+x^{-1}|A(x)| .
\end{aligned}
$$

Then the condition is

$$
I=\int_{[0, \infty)} \frac{x^{2}|d G(x)|}{U(x)+x|A(x)|}=\int_{[0, \infty)} \frac{|d G(x)|}{k(x)}<\infty .
$$

(We remark that Refs. 3, 4 and 12 use a slightly different but closely related function $h(\cdot)$ rather than $k(\cdot)$ in (1.11) and elsewhere; these are equivalent in our context, as pointed out in Ref. 6.)

Proof of Theorem 1. The result is known from Ref. 6 for $b=0$ (note that we always have $T_{r}<\infty$ a.s. for all $r>0$ in this case, as long as $X$ is not degenerate at 0 ), so take $0<b<1$. First assume (1.5). Then for $0<b \leqslant 1 / 2$, $T_{r} \stackrel{\text { a.s. }}{<} \infty$ for all $r>0$ follows from the law of the iterated logarithm or from the strong law of large numbers, according as $E X^{2}<\infty$ and $E X=0$, or $0<|E X| \leqslant E|X|<\infty$; while for $1 / 2<b<1$ it follows from the strong law 
of large numbers, under the assumption that $0<|E X| \leqslant E|X|<\infty$. Also (1.5) implies (1.4) which implies (1.8). So, replacing $n$ by $T_{r}$, we can argue that

$$
\frac{\left|X_{T_{r}}\right|}{\left|S_{T_{r}}\right|} \stackrel{\text { a.s. }}{\rightarrow} 0 \quad \text { as } r \rightarrow \infty
$$

and hence, because $\left|S_{T_{r}}\right| \leq r T_{r}^{b}+\left|X_{T_{r}}\right|$, we have for large enough $r$

$$
0<\frac{1}{r}\left(\frac{\left|S_{T_{r}}\right|}{T_{r}^{b}}-r\right) \leqslant \frac{\left|X_{T_{r}}\right|}{r T_{r}^{b}} \leqslant \frac{\left|X_{T_{r}}\right|}{\left|S_{T_{r}}\right|-\left|X_{T_{r}}\right|} \stackrel{\text { a.s. }}{\rightarrow} 0,
$$

which is (1.6). Clearly this implies (1.7), and our major task is to show that (1.7) implies (1.5), which we will do by establishing (1.11), and then arguing that this gives (1.5) for the values of $b$ specified.

So, let (1.7) hold. First note that, since $\left|S_{T_{r}-1}\right| \leqslant r T_{r}^{b}$, a consequence of (1.7) is that

$$
\limsup _{r \rightarrow \infty} \frac{\left|X_{T_{r}}\right|}{r T_{r}^{b}} \leqslant \limsup _{r \rightarrow \infty} \frac{\left|S_{T_{r}}\right|}{r T_{r}^{b}}+1 \stackrel{\text { a.s. }}{<} L+1:=K,
$$

where of course $K \in(2, \infty)$. Moreover (1.7) implies the corresponding in probability condition, so by Theorems 2.4 and 2.5 of Ref. 4 (see also Theorems $2.1-2.3$ of Ref. 3 ) we know that either $0<b<1, b \neq 1 / 2$, and $S \in R S$, or $0<b<1 / 2, \quad S \in D_{0}(N)$. In the first case it is known that either $A(x)$ is positive for all large enough $x$, or negative for all large enough $x$, that $|A(x)|$ is slowly varying at $\infty$, and that $U(x)=o(x|A(x)|)$ as $x \rightarrow \infty$; and thus that

$$
k(x)=x^{-2} U(x)+x^{-1}|A(x)| \backsim x^{-1}|A(x)|, \quad \text { as } x \rightarrow \infty .
$$

In the second case it is known that $U$ is slowly varying at $\infty$, and that $x|A(x)|=o(U(x))$ as $x \rightarrow \infty$; and thus that $k(x) \backsim x^{-2} U(x)$. Furthermore, in both cases,

$$
\frac{G(x)}{k(x)} \rightarrow 0 \quad \text { as } x \rightarrow \infty .
$$

So from now on we can assume that $k(\cdot) \in R V(-1)$ or that $0<b<1 / 2$ and $k(\cdot) \in R V(-2)$. (Here $R V(\alpha)$ is the class of positive functions which are regularly varying at $\infty$ with index $\alpha$; see Ref. 1 ). This allows us to rewrite (1.11) in a simpler form: letting

$$
J:=\int_{1}^{\infty} \frac{G(x) d x}{x k(x)}=\int_{1}^{\infty}|d G(x)| \int_{1}^{x} \frac{d y}{y k(y)},
$$

since the inner integral is asymptotic to $c / k(x)$ as $x \rightarrow \infty$ (here and throughout $c, c_{1}, c_{2}, \ldots$ denote generic positive constants whose values can 
change from one line to the next), we see that $J<\infty$ is equivalent to (1.11). Next note that for $r>0$ and $x>2 r$

$$
\begin{aligned}
P\left(\frac{\left|X_{T_{r}}\right|}{T_{r}^{b}}>x\right) & =\sum_{i \geqslant 1} P\left(T_{r}=i,\left|X_{i}\right|>x i^{b}\right) \\
& =\sum_{i \geqslant 1} P\left(\max _{1 \leqslant j<i} \frac{\left|S_{j}\right|}{j^{b}} \leqslant r<\frac{\left|S_{i}\right|}{i^{b}}, \frac{\left|X_{i}\right|}{i^{b}}>x\right) \\
& =\sum_{i \geqslant 1} P\left(\max _{1 \leqslant j<i} \frac{\left|S_{j}\right|}{j^{b}} \leqslant r, \frac{\left|X_{i}\right|}{i^{b}}>x\right)(\text { as } x>2 r) \\
& =\sum_{i \geqslant 1} G\left(x i^{b}\right) P\left(T_{r} \geqslant i\right) .
\end{aligned}
$$

To exploit this, we need some results about $P\left(T_{r} \geqslant i\right)$ and $P\left(T_{r} \leqslant i\right)$; these are proved in Refs. 3 and 4 by establishing extensions of the classical results for $b=0$ in Ref. 12. ( $\Gamma_{r}$ is defined using the $h(\cdot)$ function for $0<$ $b<1 / 2$ in Ref. 3 and extended to $0<b<1$ in Ref. 4, Section 4. Again we can equivalently use the $k(\cdot)$ function as in (1.15) and Ref. 11.)

Proposition 3. If $0<b<1$ we can define a function $\Gamma$ by

$$
\Gamma_{r}=\inf \left\{x: x k\left(r x^{b}\right) \geqslant 1\right\}, \quad r>0 .
$$

Then $\Gamma_{r} \rightarrow \infty$ as $r \rightarrow \infty$,

$$
\Gamma_{r} k\left(r \Gamma_{r}^{b}\right)=1, \quad r>0
$$

there is a $\delta \in(0,1)$ with

$$
P\left(T_{r} \leqslant \delta \Gamma_{r}\right) \leqslant 1 / 2
$$

and for any $a>0$

$$
\sum_{m=1}^{\infty} m^{a-1} P\left(T_{r} \geqslant m\right) \approx \Gamma_{r}^{a} .
$$

[Here and elsewhere $\approx$ means that the ratio of the two sides is in $\left[c_{1}, c_{2}\right]$, for some $0<c_{1}<c_{2}<\infty$, for all sufficiently large $r$.]

Another useful fact, valid in all cases, is that

$$
\frac{x^{2}}{y^{2}} \leqslant \frac{k(y)}{k(x)} \leqslant 3, \quad y \geqslant x>0 .
$$


In our particular situation, we have extra information (see Ref. 1, pp. 28-29). As the inverse of a function in $R V(1-b)$ or $R V(1-2 b)$, we have $\Gamma \in R V(1 /(1-b))$ when $k(\cdot) \in R V(-1)$, and $\Gamma \in R V(1 /(1-2 b))$ when $k(\cdot) \in$ $R V(-2)$. (Recall in this latter case that $b<1 / 2$.)

Using (1.17) in (1.14) gives, for $x>2 r>0$,

$$
\begin{aligned}
P\left(\frac{\left|X_{T_{r}}\right|}{T_{r}^{b}}>x\right) & \geqslant \frac{1}{2} \sum_{k \leqslant \delta \Gamma_{r}} G\left(x k^{b}\right) \geqslant \frac{\delta \Gamma_{r}}{2} G\left(x \delta^{b} \Gamma_{r}^{b}\right) \\
& =\frac{\delta G\left(\delta^{b} x \Gamma_{r}^{b}\right)}{2 k\left(r \Gamma_{r}^{b}\right)}
\end{aligned}
$$

where we have also used (1.16). Now write $g(r)=r \Gamma_{r}^{b}$, let $g^{-1}$ denote an asymptotic inverse of $g$; that is, $g^{-1}(x)=\inf \{r \geqslant 1: g(r)>x\}$, for large $x$, (see Ref. 1, p. 28), and put

$$
r(n)=g^{-1}\left(2^{n}\right), \quad n \geqslant 1 .
$$

Then $g \in R V(1 /(1-b))$ or $g \in R V((1-b) /(1-2 b))$, and we have $g^{-1} \in$ $R V(1-b)$ or $R V((1-2 b) /(1-b))$. Also, $g^{-1}$ is non-decreasing. So we can fix $n_{0}$ such that $r(n)$ is strictly increasing for $n \geqslant n_{0}$ and

$$
\sup _{x \geqslant 2^{n_{0}}}\left|\frac{g\left(g^{-1}(x)\right)}{x}-1\right| \leqslant \frac{1}{2} .
$$

We will also write $r(\cdot)$ for a continuous and increasing interpolant of $r$ on $\left[n_{0}, \infty\right)$, so that

$$
\frac{1}{2} \leqslant \frac{r(x) \Gamma_{r(x)}^{b}}{2^{x}} \leqslant \frac{3}{2} \text { for all } x \geqslant n_{0}
$$

and define the event

$$
E_{n}:=\left\{\frac{\left|X_{T_{r(n)}}\right|}{T_{r(n)}^{b}}>2 \operatorname{Kr}(n)\right\} .
$$

Putting $r=r(n)$ and $x=2 K r(n) / \delta^{b}>2 r(n)$ in (1.20) gives, for $n \geqslant n_{0}$,

$$
\begin{aligned}
P\left(E_{n}\right) \geqslant P\left(\frac{\left|X_{T_{r(n)}}\right|}{T_{r(n)}^{b}}>\frac{2 K r(n)}{\delta^{b}}\right) & \geqslant \frac{\delta G\left(2 K r(n) \Gamma_{r(n)}^{b}\right)}{2 k\left(r(n) \Gamma_{r(n)}^{b}\right)} \\
& \geqslant \frac{c_{1} G\left(4 K 2^{n}\right)}{k\left(2^{n}\right)}
\end{aligned}
$$


where $c_{1}>0$ and we have used (1.19). Hence

$$
\begin{aligned}
\sum_{m \geqslant n \geqslant n_{0}} P\left(E_{n}\right) & \geqslant c_{1} \sum_{m \geqslant n \geqslant n_{0}} \frac{G\left(4 K 2^{n}\right)}{k\left(2^{n}\right)} \geqslant c_{2} \sum_{m \geqslant n \geqslant n_{0}} \sum_{2^{n} \leqslant j<2^{n+1}} \frac{G(4 K j)}{j k(j)} \\
& =c_{2} \sum_{2^{m+1}>j \geqslant 2^{n_{0}}} \frac{G(4 K j)}{j k(j)} \geqslant c_{3} \int_{4 K 2^{n_{0}}}^{8 K 2^{m}} \frac{G(x) d x}{x k(x)}
\end{aligned}
$$

So if we can show that $\sum_{n \geqslant n_{0}} P\left(E_{n}\right)$ is finite, this would give $J<\infty$, thus (1.11), hence (1.4). Since we already know that either $0<b<1, b \neq 1 / 2$, and $S \in R S$, or $0<b<1 / 2$ and $S \in D_{0}(N)$, we deduce (1.5).

We reach this conclusion by contradiction, showing that the assumption

$$
\sum_{n \geqslant n_{0}} P\left(E_{n}\right)=\infty
$$

leads to $P\left(E_{n}\right.$ i.o. $)>0$, which of course would contradict (1.12). So now we assume $(1.23)$ and, to get $P\left(E_{n}\right.$ i.o. $)>0$, aim to apply the generalised Borel-Cantelli lemma in Ref. 13, p. 317. To do this we only need to establish that

$$
\limsup _{n \rightarrow \infty} \frac{\sum_{i=1}^{n} \sum_{j=i+1}^{n} P\left(E_{i} \cap E_{j}\right)}{\left(\sum_{1}^{n} P\left(E_{i}\right)\right)^{2}}<\infty .
$$

Establishing (1.24) requires some rather intricate though not routine calculations. So as not to interrupt the main points we relegate this to the Appendix, and complete this section with some comments and the application to Kesten and Maller. ${ }^{10}$

Remark 4. The argument in the first paragraph of proof of Theorem 1 in fact shows that (1.4) implies a.s. relative stability of the overshoot whenever $b \geqslant 0$ and $T_{r}<\infty$ a.s. for all $r>0$; thus, when $0<b<1$ and (1.4) holds, or when $b \geqslant 1$ and $E|X|^{1 / b}=\infty$. So we have sufficient conditions for the a.s. relative stability in all cases. As discussed in Refs. 3 and 4, for the converses, the cases $b=1 / 2, b=1$ clearly have special features and the case $b>1$ seems to present special difficulties.

Finally we point our that our result leads to an alternative proof of the following result, due to Ref. 10 . 
Proposition 5. Assume that $\frac{1}{2}<b<1, E|X|<\infty, E X=0, E\left(X^{+}\right)^{\frac{1}{b}}=$ $\infty$, and

$$
X \stackrel{\text { a.s. }}{\geqslant}-c \text { for some } 0<c<\infty \text {. }
$$

Then

$$
\limsup _{n \rightarrow \infty} \frac{S_{n}}{n^{b}} \stackrel{\text { a.s. }}{=} \infty
$$

Proof. Assuming that $E|X|^{\frac{1}{b}}=\infty$, the Markinkiewicz-Zygmund strong law of large numbers (see Ref. 2, p. 125) gives that

$$
\limsup _{n \rightarrow \infty} \frac{\left|S_{n}\right|}{n^{b}} \stackrel{\text { a.s. }}{=} \infty
$$

so if (1.26) failed, we would have

$$
\limsup _{n \rightarrow \infty} \frac{S_{n}}{n^{b}} \stackrel{\text { a.s. }}{<} \infty \text { and } \quad \liminf _{n \rightarrow \infty} \frac{S_{n}}{n^{b}} \stackrel{\text { a.s. }}{=}-\infty
$$

This would then imply that

$$
\lim _{r \rightarrow \infty} X_{T_{r}} \stackrel{\text { a.s. }}{<} 0
$$

In view of (1.25) this would imply that $\left|X_{T_{r}}\right|=-X_{T_{r}}=O\left(r T_{r}^{b}\right)$, a.s., hence $\left|S_{T_{r}}\right|=O\left(r T_{r}^{b}\right)$, a.s., as $r \rightarrow \infty$, and so (1.7) would hold, giving a.s. relatively stability of the overshoot. But this is equivalent to (1.5), contradicting our assumptions, and proving our claim.

Remark 6. Actually it is shown in Ref. 10 that the Proposition holds without the assumption (1.25). But the result of Proposition 5 as we state it provides a key step in the proof in Ref. 10 .

\section{APPENDIX: PROOF OF (1.24)}

In the case $b=0$ it is possible to find an upper bound for $P\left(E_{n}\right)$ which differs from the lower bound only by a constant multiple; when $b>0$ things are a little more complicated. We proceed as follows. From 
(1.14) and (1.18), for $r$ large enough and $x>2 r$,

$$
\begin{aligned}
P\left(\frac{\left|X_{T_{r}}\right|}{T_{r}^{b}}>x\right) & \leqslant \sum_{i \leqslant \Gamma_{r}} G\left(x i^{b}\right)+G\left(x \Gamma_{r}^{b}\right) \sum_{i>\Gamma_{r}} P\left(T_{r} \geqslant i\right) \\
& \leqslant \sum_{i \leqslant \Gamma_{r}} G\left(x i^{b}\right)+c_{1} G\left(x \Gamma_{r}^{b}\right) \Gamma_{r} \leq\left(1+c_{1}\right) \sum_{i \leqslant \Gamma_{r}} G\left(x i^{b}\right) .
\end{aligned}
$$

Put $r=r(n)$ and $x=2 \operatorname{Kr}(n)>2 r(n)$ in this to get

$$
P\left(E_{n}\right) \leqslant c_{2} \sum_{i \leqslant \Gamma_{r(n)}} G\left(2 K r(n) i^{b}\right):=c_{2} \varepsilon_{n}, \quad n \geqslant n_{0} .
$$

Now note that

$$
\begin{aligned}
\sum_{i=n_{0}}^{n} \varepsilon_{i} & =\sum_{i=n_{0}}^{n} \sum_{m \leqslant \Gamma_{r(i)}} G\left(2 K r(i) m^{b}\right) \leqslant \sum_{i=n_{0}}^{n} \int_{z=0}^{\Gamma_{r(i)}} G\left(2 K r(i) z^{b}\right) d z \\
& =\frac{1}{b} \sum_{i=n_{0}}^{n}(2 K r(i))^{-1 / b} \int_{y=0}^{2 K r(i) \Gamma_{r(i)}^{b}} y^{\frac{1-b}{b}} G(y) d y \\
& \leqslant c_{1} \int_{n_{0}}^{n}(r(u))^{-1 / b} \int_{y=0}^{4 K 2^{u}} y^{\frac{1-b}{b}} G(y) d y d u \\
& =c_{1} \int_{y=0}^{c_{2} 2^{n}} y^{\frac{1-b}{b}} G(y) d y \int_{n \geqslant u \geqslant n_{0}, 2^{u} \geqslant y / c_{2}}(r(u))^{-1 / b} d u \\
& \leqslant c_{3} \int_{y=0}^{c_{2} 2^{n}} y^{\frac{1-b}{b}} G(y) d y \int_{n \geqslant u \geqslant n_{0}, 2^{u} \geqslant y / c_{2}} \frac{d u}{2^{\frac{u}{b}} k\left(2^{u}\right)} \\
& \leqslant c_{4} \int_{y=0}^{c_{2} 2^{n}} y^{\frac{1-b}{b}} G(y) d y \int_{y / c_{2}}^{\infty} \frac{d v}{v^{\frac{1+b}{b}} k(v)} .
\end{aligned}
$$

Noting that the integrand is regularly varying with index strictly less than -1 , we see that

$$
\int_{y / c_{2}}^{\infty} \frac{d v}{v^{\frac{1+b}{b}} k(v)} \backsim \frac{c_{5}}{y^{\frac{1}{b}} k(y)} \quad \text { as } y \rightarrow \infty
$$

and hence

$$
\sum_{i=n_{0}}^{n} \varepsilon_{i} \leqslant c_{6} \int_{0}^{c_{2} 2^{n}} \frac{y^{\frac{1-b}{b}} G(y)}{y^{\frac{1}{b}} k(y)} d y=c_{6} \int_{0}^{c_{2} 2^{n}} \frac{G(y)}{y k(y)} d y .
$$


Going back to (2.1) and writing $V(x)=\int_{0}^{x} \frac{G(y)}{y k(y)} d y$, we see that

$$
\sum_{i=n_{0}}^{n} P\left(E_{i}\right) \leqslant c_{7} V\left(c_{2} 2^{n}\right), n \geqslant n_{0}
$$

and because we are assuming that the lefthand side tends to $\infty$ as $n \rightarrow \infty$, we have

$$
\int_{0}^{\infty} \frac{G(y)}{y k(y)} d y=\infty
$$

Since

$$
\frac{x V^{\prime}(x)}{V(x)}=\frac{G(x)}{V(x) k(x)} \rightarrow 0,
$$

we see that $V \in R V(0)$, and we conclude from (1.22) and (2.3) that

$$
\sum_{i=n_{0}}^{n} P\left(E_{i}\right) \approx V\left(2^{n}\right) .
$$

Now take $j>i \geqslant n_{0}$ fixed and write $P\left(E_{i} \cap E_{j}\right)=P_{i, j}+Q_{i, j}$, where $P_{i, j}=$ $P\left(E_{i} \cap E_{j}, T_{r(i)}<T_{r(j)}\right)$ and $Q_{i, j}=P\left(E_{i} \cap E_{j}, T_{r(i)}=T_{r(j)}\right)$. Note that, without loss of generality, we can take $K$ as large as we wish; initially we have $2 K>4$, so can write

$$
\begin{aligned}
P_{i, j} & =\sum_{m=1}^{\infty} \sum_{l=m+1}^{\infty} P\left(E_{i} \cap E_{j}, T_{r(i)}=m, T_{r(j)}=l\right) \\
& =\sum_{m=1}^{\infty} \sum_{l=m+1}^{\infty} P\left(A_{m, i} \cap B_{l, j}\right), \text { say }
\end{aligned}
$$

where (cf. (1.14))

$$
A_{m, i}=\left\{\max _{1 \leqslant v<m} \frac{\left|S_{v}\right|}{v^{b}} \leqslant r(i), \frac{\left|X_{m}\right|}{m^{b}}>2 \operatorname{Kr}(i)\right\}
$$

and

$$
B_{l, j}=\left\{\max _{1 \leqslant v<l} \frac{\left|S_{v}\right|}{v^{b}} \leqslant r(j), \frac{\left|X_{l}\right|}{l^{b}}>2 K r(j)\right\} .
$$

Next, we split this sum and write $P_{i, j}=P_{i, j}^{(1)}+P_{i, j}^{(2)}$, where

$$
P_{i, j}^{(1)}=\sum_{m=1}^{\infty} \sum_{1 \leqslant t<m^{*}} P\left\{A_{m, i} \cap B_{m+t, j}\right\},
$$


$\Delta>0$ is a fixed positive constant, and $m^{*}$ denotes the integer part of $m \Delta$. Note that if $B_{m+t, j}$ occurs for a value of $t$ with $1 \leqslant t<m^{*}$, then

$$
\left|S_{m+v}\right| \leqslant r(j)(m+v)^{b} \leqslant r(j)(1+\Delta)^{b} m^{b} \quad \text { for } v=0,1, \ldots, t-1,
$$

also $\left|S_{m}\right| \leqslant r(j) m^{b}$. So if we write $\hat{S}_{v}=S_{m+v}-S_{m}$, which is independent of $A_{m, i}$ for $v=0,1, \ldots$, then we have

$$
\max _{1 \leqslant v<t}\left|\hat{S}_{v}\right| \leqslant r(j)(1+\Delta)^{b} m^{b}+r(j) m^{b} \leqslant K r(j) m^{b},
$$

as long as we take $K$ large enough that $(1+\Delta)^{b}+1 \leqslant K$. Thus, we have, for $l>m$,

$$
\begin{aligned}
& \sum_{1 \leqslant t \leqslant m^{*}} P\left(B_{m+t, j} \mid A_{m, i}\right) \\
& \leqslant \sum_{1 \leqslant t \leqslant m^{*}} P\left(\max _{1 \leqslant v<t}\left|\hat{S}_{v}\right| \leqslant K r(j) m^{b},\left|X_{m+t}\right|>2 K r(j) m^{b}\right) \\
& \left.=\sum_{1 \leqslant t \leqslant m^{*}} P\left(T^{(0)}\left(\operatorname{Kr}(j) m^{b}\right)\right)=t,\left|X_{t}\right|>2 K r(j) m^{b}\right) \\
& \leqslant P\left(T^{(0)}\left(K r(j) m^{b}\right) \leqslant m^{*}\right),
\end{aligned}
$$

where we have written $T^{(0)}(r)$ for $T_{r}$ in the case $b=0$. For this we have, from Ref. 12,

$$
P\left(T^{(0)}\left(K r(j) m^{b}\right) \leqslant m^{*}\right) \leqslant c m^{*} k\left(K r(j) m^{b}\right) .
$$

Next we show that

$$
\sum_{j=i+1}^{\infty} k\left(K r(j) m^{b}\right) \leqslant c k\left(r(i) m^{b}\right) \quad \text { for } m \geqslant 1 \text { and } i \geqslant n_{0} .
$$

We have $r(n+1) / r(n) \rightarrow \gamma$, where $\gamma=2^{1-b}$ (in case $\left.b<1\right)$ or $2^{(1-2 b) /(1-b)}$ (in case $b<1 / 2$ ), so $1<\gamma<2$. Thus, without loss of generality we can assume that

$$
1<\gamma_{1} \leqslant \frac{r(n+1)}{r(n)} \leqslant \gamma_{2}<2 \text { for all } n \geqslant n_{0} .
$$


So when $j \geqslant i \geqslant n_{0}$ we have $r(j) \geqslant r(i) \gamma_{1}^{j-i}$, and hence by (1.19), as $i \rightarrow \infty$,

$$
\begin{aligned}
\sum_{j=i+1}^{\infty} k\left(K r(j) m^{b}\right) & \leqslant 3 \sum_{j=i+1}^{\infty} k\left(K r(i) \gamma_{1}^{j-i} m^{b}\right) \\
& \leqslant c \int_{0}^{\infty} k\left(K r(i) \gamma_{1}^{x} m^{b}\right) d x=c \int_{1}^{\infty} k\left(K r(i) y m^{b}\right) \frac{d y}{y} \\
& =c \int_{K r(i) m^{b}}^{\infty} k(z) \frac{d z}{z} \sim c k\left(K r(i) m^{b}\right) \sim c k\left(r(i) m^{b}\right)
\end{aligned}
$$

(recall that $k(\cdot) \in R V(\alpha)$ with $\alpha=-1$ or -2 ), and (2.6) follows.

Thus, since $P\left(A_{m, i}\right)=P\left(T_{r(i)} \geqslant m\right) G\left(2 K r(i) m^{b}\right)$, and $2^{i-1} \leqslant r(i) \Gamma_{r(i)}^{b} \leqslant$ $3.2^{i-1}$, we have the bound

$$
\begin{aligned}
\sum_{j=i+1}^{n} P_{i, j}^{(1)} \leqslant & c_{1} \sum_{m=1}^{\infty} m P\left(T_{r(i)} \geqslant m\right) G\left(2 K r(i) m^{b}\right) k\left(r(i) m^{b}\right) \\
\leqslant & c_{1} \sum_{m \leqslant \Gamma_{r(i)}} m G\left(2 K r(i) m^{b}\right) k\left(r(i) m^{b}\right) \\
& +3 c_{1} G\left(2 K r(i) \Gamma_{r(i)}^{b}\right) k\left(r(i) \Gamma_{r(i)}^{b}\right) \sum_{m>\Gamma_{r(i)}} m P\left(T_{r(i)} \geqslant m\right) \\
= & c_{1}(\sigma(1, i)+\sigma(2, i)), \text { say }
\end{aligned}
$$

(where we used (1.19) in the second inequality). Now by (1.18) with $a=2$ we have

$$
\begin{aligned}
\sigma(2, i) & \leq 2 c_{2} G\left(2 K r(i) \Gamma_{r(i)}^{b}\right) k\left(r(i) \Gamma_{r(i)}^{b}\right) \Gamma_{r(i)}^{2} \\
& \leq 6 c_{2} \sigma(1, i) \text { (using (1.19) again), }
\end{aligned}
$$

so we need only consider $\sigma(1, i)$. By repeating the calculation leading to (2.2) we see that

$$
\sum_{i=n_{0}}^{n} \sigma(1, i) \leqslant c_{4} \int_{y=0}^{c_{2} 2^{n}} y^{\frac{2-b}{b}} G(y) k(y) d y \int_{y / c_{2}}^{\infty} \frac{d v}{v^{\frac{2+b}{b}} k^{2}(v)}
$$

Again the integrand is regularly varying with index strictly less than -1 , so it follows that

$$
\int_{y / c_{2}}^{\infty} \frac{d v}{v^{\frac{2+b}{b}} k^{2}(v)} \backsim \frac{c_{5}}{y^{\frac{2}{5}} k^{2}(y)} \quad \text { as } y \rightarrow \infty
$$


and hence

$$
\sum_{i=n_{0}}^{n} \sigma(1, i) \leqslant c_{6} \int_{y=0}^{c_{2} 2^{n}} \frac{y^{\frac{2-b}{b}} G(y)}{y^{\frac{2}{b}} k(y)} d y=c_{6} V\left(c_{2} 2^{n}\right) .
$$

From (2.4) we now deduce

$$
\limsup _{n \rightarrow \infty} \frac{\sum_{i=1}^{n} \sum_{j=i+1}^{n} P_{i, j}^{(1)}}{\left(\sum_{1}^{n} P\left(E_{i}\right)\right)^{2}}<\infty .
$$

We can also write

$$
P_{i, j}^{(2)}=\sum_{m=1}^{\infty} \sum_{s=1}^{\infty} P\left\{A_{m, i} \cap B_{m+m^{*}+s, j}\right\} .
$$

Again write $\hat{S}_{v}=S_{m+v}-S_{m}$ and now take $K \geqslant(2+\Delta)((1+\Delta) / \Delta)^{b}$. Then

$$
\begin{aligned}
& \sum_{s=1}^{\infty} P\left(B_{m+m^{*}+s, j} \mid A_{m, i}\right) \\
& \quad \leqslant \sum_{s=1}^{\infty} P\left(\max _{-m^{*}<v<s} \frac{\left|\hat{S}_{m^{*}+v}\right|}{\left(m+m^{*}+v\right)^{b}} \leqslant 2 r(j), \frac{\left|X_{m+m^{*}+s}\right|}{\left(m+m^{*}+s\right)^{b}}>2 K r(j)\right) \\
& \quad \leqslant \sum_{s=1}^{\infty} P\left(\max _{-m^{*}<v<s} \frac{\left|\hat{S}_{m^{*}+v}\right|}{\left(m^{*}+v\right)^{b}} \leqslant 2\left(\frac{1+\Delta}{\Delta}\right)^{b} r(j), \frac{\left|X_{m+m^{*}+s}\right|}{\left(m+m^{*}+s\right)^{b}}>2 K r(j)\right) \\
& \leqslant \sum_{t=m^{*}+1}^{\infty} P\left(\max _{v<t} \frac{\left|\hat{S}_{v}\right|}{v^{b}} \leqslant K r(j), \frac{\left|X_{m+t}\right|}{(m+t)^{b}}>2 K r(j)\right) \\
& \leqslant P\left(\left|X\left(T_{K r(j)}\right)\right|>2 K r(j) T_{K r(j)}^{b}\right)=P\left(E_{j}^{\prime}\right), \text { say. }
\end{aligned}
$$

It follows that

$$
P_{i, j}^{(2)} \leqslant \sum_{m=1}^{\infty} P\left(A_{m, i}\right) P\left(E_{j}^{\prime}\right)=P\left(E_{i}\right) P\left(E_{j}^{\prime}\right) .
$$

By analogous calculations to (2.1) and (2.2), we can check that

$$
\sum_{n_{0}}^{n} P\left(E_{j}^{\prime}\right) \leq c \sum_{n_{0}}^{n} P\left(E_{j}\right) \text {. }
$$

Thus

$$
\limsup _{n \rightarrow \infty} \frac{\sum_{i=1}^{n} \sum_{j=i+1}^{n} P_{i, j}^{(2)}}{\left(\sum_{1}^{n} P\left(E_{i}\right)\right)^{2}}<\infty .
$$


Finally, we have to deal with $Q_{i, j}=P\left(E_{i} \cap E_{j}, T_{r(i)}=T_{r(j)}\right), j>i \geqslant n_{0}$. We can write

$$
\begin{aligned}
Q_{i, j} & =\sum_{m=1}^{\infty} P\left(\max _{v<m} \frac{\left|S_{v}\right|}{v^{b}} \leqslant r(i),\left|X_{m}\right|>2 K r(j) m^{b}\right) \\
& =\sum_{m=1}^{\infty} P\left(\max _{v<m} \frac{\left|S_{v}\right|}{v^{b}} \leqslant r(i)\right) G\left(2 K r(j) m^{b}\right) \\
& \leqslant \sum_{m=1}^{\Gamma_{r(i)}} G\left(2 K r(j) m^{b}\right)+G\left(2 K r(j) \Gamma_{r(i)}^{b}\right) \sum_{m>\Gamma_{r(i)}} P\left(T_{r(i)} \leqslant m\right) \\
& \leqslant \sum_{m=1}^{\Gamma_{r(i)}} G\left(2 K r(j) m^{b}\right)+c \Gamma_{r(i)} G\left(2 K r(j) \Gamma_{r(i)}^{b}\right) \quad(\text { using }(1.18)) \\
& \leq(1+c) \sum_{m=1}^{\Gamma_{r(i)}} G\left(2 K r(j) m^{b}\right) .
\end{aligned}
$$

Recall that when $j \geqslant i \geqslant n_{0}$ we have $r(j) \geqslant r(i) \gamma_{1}^{j-i}$; thus

$$
Q_{i, j} \leqslant(1+c) \sum_{m=1}^{\Gamma_{r(i)}} G\left(2 K \gamma_{1}^{j-i} r(i) m^{b}\right) .
$$

At this stage we need the following technical fact;

Lemma 7. Define

$$
W(x)=\int_{x}^{\infty} \frac{G(y)}{y} d y .
$$

Then if $S \in R S$ or $S \in D_{0}(N)$ we have, for some $c>0$,

$$
\int_{1}^{x} \frac{W(y)}{y k(y)} d y \leqslant c V(x) \text { for all sufficiently large } x .
$$

Proof of Lemma 7. First consider the case that $S \in D_{0}(N)$, when

$$
k(x) \sim \frac{U(x)}{x^{2}}, \text { where } U(x)=\int_{0}^{x} 2 y G(y) d y
$$

and we have

$$
U \in R V(0) \text { and } \quad \frac{x^{2} G(x)}{U(x)} \rightarrow 0 \text { as } x \rightarrow \infty .
$$


Thus

$$
\begin{aligned}
2 W(x) & =\int_{x}^{\infty} \frac{2 y G(y)}{y^{2}} d y=\int_{x}^{\infty} \frac{U^{\prime}(y)}{y^{2}} d y \\
& =-\frac{U(x)}{x^{2}}+2 \int_{x}^{\infty} \frac{U(y)}{y^{3}} d y=o\left(\frac{U(x)}{x^{2}}\right) .
\end{aligned}
$$

Now

$$
\int_{1}^{x} \frac{W(y)}{y k(y)} d y \backsim \int_{1}^{x} \frac{y W(y)}{U(y)} d y
$$

and

$$
\int_{1}^{x} \frac{G(y)}{y k(y)} d y \backsim \int_{1}^{x} \frac{y G(y)}{U(y)} d y, \quad \text { as } x \rightarrow \infty .
$$

But

$$
\begin{aligned}
\int_{1}^{x} \frac{y G(y)}{U(y)} d y & =\int_{1}^{x} \frac{G(y)}{y} \frac{y^{2}}{U(y)} d y=-\int_{1}^{x} W^{\prime}(y) \frac{y^{2}}{U(y)} d y \\
& =\frac{W(1)}{U(1)}-\frac{x^{2} W(x)}{U(x)}+\int_{1}^{x} \frac{W(y)}{U^{2}(y)}\left\{2 y U(y)-y^{2} U^{\prime}(y)\right\} d y \\
& =O(1)+2 \int_{1}^{x} \frac{y W(y)}{U^{2}(y)}\left\{U(y)-y^{2} G(y)\right\} d y \\
& =O(1)+(2+o(1)) \int_{1}^{x} \frac{y W(y)}{U(y)} d y
\end{aligned}
$$

and the result follows.

If $S \in R S$ we have $A(x)>0$ for all large enough $x$, or $A(x)<0$ for all large enough $x$, and $|A(x)|$ is in $R V(0)$; and we also have $U(x)=o(x A(x))$ as $x \rightarrow \infty$. So, taking $A(x)>0$ for all large enough $x$, we have

$$
\begin{aligned}
2 W(x) & =\int_{x}^{\infty} \frac{2 y G(y)}{y^{2}} d y=\int_{x}^{\infty} \frac{U^{\prime}(y)}{y^{2}} d y \\
& =-\frac{U(x)}{x^{2}}+2 \int_{x}^{\infty} \frac{U(y)}{y^{3}} d y \\
& =o\left(\frac{A(x)}{x}+2 \int_{x}^{\infty} \frac{A(y)}{y^{2}} d y\right)=o\left(\frac{A(x)}{x}\right) .
\end{aligned}
$$


If we now observe that

$$
\begin{aligned}
\int_{1}^{x} \frac{G(y)}{A(y)} d y & =\int_{1}^{x} \frac{G(y)}{y} \frac{y}{A(y)} d y=-\int_{1}^{x} W^{\prime}(y) \frac{y}{A(y)} d y \\
& =\frac{W(1)}{A(1)}-\frac{x W(x)}{A(x)}+\int_{1}^{x} \frac{W(y)}{A(y)}\left\{1-\frac{y A^{\prime}(y)}{A(y)}\right\} d y \\
& =O(1)+(1+o(1)) \int_{1}^{x} \frac{W(y)}{A(y)} d y,
\end{aligned}
$$

because $\left(y A^{\prime}(y)\right) / A(y) \rightarrow 0$ as $y \rightarrow \infty$, then this case is also proven. A similar proof works if $A(x)<0$ for all large enough $x$.

Using Lemma 7, we can argue that

$$
\begin{aligned}
& \sum_{i=1}^{n} \sum_{j=i+1}^{n} \sum_{m=1}^{\Gamma_{r(i)}} G\left(2 K \gamma_{1}^{j-i} r(i) m^{b}\right) \\
& \quad \leqslant \int_{x=0}^{n} \int_{y=0}^{\infty} \int_{w=0}^{r(x) \Gamma_{r(x)}^{b}} w^{\frac{1-b}{b}} r(x)^{-\frac{1}{b}} G\left(2 K \gamma_{1}^{y} w\right) d w d y d x \\
& \quad \leqslant c \int_{x=0}^{n} \int_{y=0}^{\infty} \int_{w=0}^{3.2^{x-1}} \frac{w^{\frac{1-b}{b}} G\left(2 K \gamma_{1}^{y} w\right)}{2^{\frac{x}{b}} k\left(2^{x}\right)} d w d y d x \\
& \quad=c \int_{v=1}^{2^{n}} \int_{w=0}^{3 . v / 2} \int_{z=1}^{\infty} \frac{w^{\frac{1-b}{b}} G(2 K z w)}{z v^{\frac{1+b}{b}} k(v)} d z d w d v \\
& \quad=c \int_{v=1}^{2^{n}} \int_{w=0}^{3 . v / 2} \frac{w^{\frac{1-b}{b}} W(2 K w)}{v^{\frac{1+b}{b}} k(v)} d w d v \quad(\mathrm{using}(2.10)) \\
& \leqslant c \int_{w=0}^{3.2^{n-1}} w^{\frac{1-b}{b}} W(2 K w) d w \int_{v=2 w / 3}^{\infty} \frac{d v}{v^{\frac{1+b}{b}} k(v)} \\
& \leqslant c \int_{w=0}^{3.2^{n-1}} \frac{W(2 K w)}{w k(w)} d w \\
& \leqslant c V\left(3.2^{n}\right) \quad(\mathrm{using}(2.11)) \\
& \sim c V\left(2^{n}\right), \quad \text { as } n \rightarrow \infty .
\end{aligned}
$$

Together with (2.7) and (2.8), this concludes the proof of (1.24), and the theorem is proved.

\section{ACKNOWLEDGMENTS}

We are grateful to a referee for a careful reading of the paper and helpful suggestions. This work is partially supported by ARC Grant DP0210572. 


\section{REFERENCES}

1. Bingham, N. H., Goldie, C. M., and Teugels, J. L. (1987). Regular Variation, Cambridge University Press, Cambridge.

2. Chow, Y. S., and Teicher, H. (1988). Probability Theory. Independence, Interchangeability, Martingales, 2nd ed. Springer Texts in Statistics, Springer-Verlag, New York, Berlin.

3. Doney, R. A., and Griffin, P. S. (2003). Overshoots over curved boundaries. Adv. Appl. Probab. 35, 417-448.

4. Doney, R. A., and Griffin, P. S. (2004). Overshoots over curved boundaries, II. Adv. Appl. Probab. 36, 1148-1174.

5. Doney, R. A., and Maller, R. A. (2000). Random walks crossing curved boundaries; stability and asymptotic distributions for exit times and positions. Adv. Appl. Probab. 32, $1117-1149$.

6. Griffin, P. S., and Maller, R. A. (1998). On the rate of growth of the overshoot and the maximum partial sum. Adv. Appl. Probab. 30, 181-196.

7. Griffin, P. S., and McConnell, T. R. (1992). On the position of a random walk at the time of first exit from a sphere. Ann.Probab., 20, 825-854.

8. Griffin, P. S., and McConnell, T. R. (1994). Gambler's ruin and the first exit position of a random walk from large spheres. Ann. Probab. 22, 1429-1472.

9. Griffin, P. S., and McConnell, T. R. (1995). $L^{p}$-boundedness of the overshoot in multidimensional renewal theory. Ann. Probab. 23, 2022-2056.

10. Kesten, H., and Maller, R. A. (1998). Random walks crossing power law boundaries. Studia Scientiarum Math. Hungarica, 34, 219-252.

11. Kesten, H., and Maller, R. A., (1998). Random walks crossing high level curved boundaries. J. Theor. Probab. 11, 1019-1074.

12. Pruitt, W. E. (1981). The growth of random walks and Lévy processes. Ann. Probab. 9, 948-956.

13. Spitzer, F. (1976). Principles of Random Walk, 2nd ed. Springer-Verlag, New York. 